\section{Imagined worlds: visions of theoretical physics}

\section{David Bohm}

Imagery in Scientific Thought: Creating 20th Century Physics.

By Arthur I. Miller.

Birkhäuser:1985. Pp.320. \$24.95.

THE ultimate origin of creative developments in science, art and in other areas of life will probably remain a mystery, no matter how far our knowledge may progress in the future. Nevertheless, it seems reasonable to suppose that a great deal of light can be shed on the subject by a careful analysis of the process in which new ideas develop in actual historical casestudies. In Imagery in Scientific Thought Arthur Miller does this for the field of science, tracing the relationship of creative thinking and the construction of new scientific concepts from pre-scientific knowledge. To this end, he focuses on the work of Poincaré, Einstein, Boltzmann, Bohr and Heisenberg, because these physicists were specifically influenced in their research by their consideration of the nature of thinking itself.

In the book, Miller makes the key point that the major change that took place through the work of these scientists was the development of a fresh attitude towards mathematics and the imagination. Thus, Poincare's view on the subject was that Euclidean geometry arose necessarily from man's earliest thought because it was needed for his survival. For Poincaré this implied the basically conventional nature of the axioms of geometry. To obtain real content, they have to be enriched with lower-level physical hypotheses. The introduction of such hypotheses is through invention, and this comes out of an intuition that cannot be defined explicitly but must be felt. This is a kind of Baconian inductivism with gaps supplied by intuitively guided inventions. Physical theories then express in the most convenient manner the "true relations between real objects" along with the invariant laws pertaining in these relationships.

Einstein adopted much of Poincaré's approach, and explicitly expressed his debt to Poincaré in calling attention to the need to "pay strict attention to the relation of reality and our concepts". However, he rejected Poincaré's a priori organizing principles along with his closely related assumption of a fundamental role in our thinking for Euclidean concepts. Rather, he regarded concepts in general as admissible "only to the extent that observable facts can be assigned to them without ambiguity". Thus, for Einstein, the question of whether Euclidean geometry is valid was basically physical (for example, that of whether it is possible to attach the Euclidean concept of length to a quasirigid measuring rod in an unambiguous way).

Einstein considered science to be essentially a refinement of everyday thinking, in which images are of course involved. $\mathrm{He}$ felt it necessary to analyse serious thinking (in contrast to "dreaming") as a series of such images ordered by a recurrent image that constantly turns up. Such an ordering element can relate many different series of hitherto unconnected elements, and this ordering element he regarded as a concept.

Though he seems to have started from Poincaré's approach of building a theory step by step by posing hypotheses to explain the experimental data, Einstein realized by the end of 1904 that the "known facts" were too restricted to solve the problem of an electromagnetic world picture. Instead he shifted to an appeal to general axioms and theories of principle, similar in structure to those of thermodynamics. These were the principle of relativity of motion and the principle of the invariance of the speed of light. Here, Einstein's thought was affected by the views of Boltzmann. For Boltzmann, our mental pictures, which he regarded as abstractions from objects actually perceived, must be clearly and unambiguously imaginable. A striking example of this is Einstein's highly imaginative picture of an observer who moves along with a light wave and sees only a static configuration of fields. The implication of this picture violated Einstein's physical intuition that the electromagnetic field is inherently dynamic, and eventually led to his taking the invariance of the speed of light as a basic principle.

Through the consideration of the properties of dynamos (as well as in other ways) Einstein was then led to regard Lorentz's idea of a local time in terms of which Maxwell's equations were invariant (to first order in $v / c$ ) as similarly indicating a general principle, which was the relativity of simultaneity (and which held to all orders in $v / c$ ). Here, Einstein was clearly guided by the notion that our ordinary concepts of time can no longer be related unambiguously to the experimental facts when we consider the full implications of electrodynamics (as he showed with the aid of various hypothetical experiments). So he was ready to go against ordinary intuitive experience when he felt this to be necessary, but he still insisted that concepts have to be some kind of abstractions from our pre-scientific perceptual experience which must be unambiguous in the senses indicated above.

This brings us to the contributions of Bohr and Heisenberg. Their analyses of the meaning of the quantum theory indicated to them that there are limitations on the possibility of unambiguous intuitive visualizability of the properties of matter at the quantum level. Bohr emphasized the need to separate the space-time and the causal descriptions. This implied that the ordinary imaginative pictures can be retained, but that they have to be ambiguous$l y$ related to experimental facts. Here, perhaps, is the fundamental difference between Bohr and Einstein, who, as we recall, insisted on the need for the possibility of an unambiguous relationship between concepts and the experimental facts. In my opinion, it is unfortunate that Miller does not bring this point out in the book, as it is of crucial importance in understanding the split that eventually came about between Bohr and Einstein, a split that profoundly affected the development of quantum theory and relativity.

Heisenberg's initial approach was, in contrast to that of Bohr, to insist that our customary intuitive visualization (Anschauung) could not be extended into the atomic domain at all. Miller follows the further development of Heisenberg's approach in some detail, to show how it led eventually to a new notion of intuition and imagination, which Heisenberg called Anschaulichkeit. This referred not to ordinary visualization, but rather to the imaginative display of the meaning of the mathematical formalism (which latter was by then regarded as the most direct and fundamental way possible of putting the truth about nature). This mode of visualization is now most commonly encountered in the Feynman diagrams which do not correspond to reflections of actual physical processes, but which serve primarily to enable physicists to think imaginatively about the meaning of the mathematics. As Heisenberg himself said, this is essentially an extension of the Platonic (and perhaps Pythagorean) approach. It may be summed up in the dictum of Jeans : "God is a mathematician".

If one looks at how modern theoretical physics is actually done, one can see that Heisenberg's approach now dominates, not only in particle physics but also in cosmology. Miller's book provides important insight into how the whole way of doing theoretical physics thus altered radically in a comparatively short time. This insight could, however, perhaps have been extended if the author had brought out, even if only briefly, how several centuries ago a similar change occurred, culminating in the Newtonian approach. To have done this would have indicated that what constitutes commonly accepted scientific practice has constantly been changing, and that it may therefore change again in an equally fundamental way. Tacitly to regard the currently accepted way of doing physics as the last word on the subject is to encourage the same sort of absolutist conclusions that took hold of the Newtonian way of doing physics, and thus help to ossify the current approach too.

The latter part of the book is a survey of various attempts of modern psychologists to account for the creative development of modern physics. It begins with a description of Wertheimer's application of Gestalt 
psychology to Einstein's process of thinking in the work that led to the theory of relativity (Wertheimer's work drew upon actual discussions with Einstein himself). Miller then goes on to a broader discussion in terms of new researches based on extensions of Piaget's work on genetic epistemology. Two different versions of genetic epistemology are considered here, the first due to Pylyshin and the second to Kosslyn. Pylyshin and Kosslyn are in agreement in that they develop detailed accounts of how imaginative thought actually proceeds and try to support these accounts on the basis of actual case-studies. Their principal disagreement is on the dynamic role of images. Roughly speaking, Kosslyn assigns a positive predictive power to images, while Pylyshin regards them, in effect, as little more than passive displays of abstract propositional thought.

Although Miller gives what seems to be a fair treatment of this whole subject, I feel that he fails to be adequately critical in that he accepts an assumption that seems to be taken for granted in all the work on genetic epistemology. This is that creativity is primarily to be understood through the development of knowledge, enriched perhaps from time to time by invention whose source is (along the lines indicated by Poincaré) ultimately in a mysterious intuition. I would like to suggest that while all of these factors are involved in creativity, its primary source is in perception, not only through the senses, but, even more, through the mind (as indicated by the Greek noesis and the German Vernunft, which latter means intuitive or perceptive reason). Kostler gives an example of this, in the sudden realization of Archimedes that the volume of water displaced by a body is the same as the volume of the body independently of the complex details of its shape. Perhaps we could say that Einstein must have similarly realized somehow that the problem with electrodynamics was essentially the same as with thermodynamics; that is, to find principles that are independent of a detailed dynamical description of all the complex processes that are actually at work in concrete experimental situations. It seems to me that a study of the history of the subject from such a perspective is necessary, if we are to have a proper account of how the mind gets out of the "rut" of thinking in terms of old concepts that have long been taken for granted.

I feel that in spite of certain inadequacies Professor Miller has made a valuable contribution to our understanding of creativity in twentieth-century physics. His book is well worth reading by all those who are interested in the history and philosophy of science, or in the understanding of the nature of creativity and its relationship to the process of thought.

David Bohm is Emeritus Professor of Theoretical Physics at Birkbeck College, University of London.

\section{From Cambridge to Moscow}

\section{Nevill Mott}

Kapitza, Rutherford, and the Kremlin.

By Lawrence Badash.

Yale University Press: 1985. Pp.129. \$20, $£ 20$.

LAWRENCE Badash has dedicated this book to the memories of Ernest Rutherford (1871-1937) and Peter Kapitza (18941984). The story of the relationship between the two men is well-known. Kapitza, a brilliant young pupil of A.F. Joffe in Leningrad, lost his wife and two children in the influenza epidemic of 1921 , and, in an effort to rescue him from his deep grief, friends arranged a trip abroad. They were successful; Kapitza knew where he wanted to go and arrived that year in Rutherford's Cavendish Laboratory. The Mond Laboratory was built for him and a Royal Society chair became his. There he developed equipment for producing strong magnetic fields and low temperatures, work on which was to culminate in the award of a Nobel Prize in 1978. The friendship between the two men must have been very deep, particularly as these branches of physics were far away from Rutherford's beloved nucleus.

Kapitza, as I remember, used to boast that he was the only Soviet citizen whose passport was endorsed for unlimited travel in and out of the Soviet Union. In 1934 he went with his second wife Anna to the congress in Leningrad which celebrated the one-hundreth anniversary of the birth of Mendeleef. They took their car to Bergen, and motored through Norway and Finland. I remember this, as I was invited to the conference, probably because I had helped Frenkel with the English version of his book on liquids, and Kapitza suggested that my wife and I should join them in their car (in the event there seemed not to be enough room for us and the luggage, and we went by boat). Once there, Kapitza was told that his country needed him and he must stay. Anna went back to Cambridge, joining him with the children more than a year later.
In the book Badash tells the story of Rutherford's efforts to help him, reproduces many of Kapitza's agonized letters to his wife and to Rutherford, and records how eventually much of the Cambridge equipment was bought by the Soviet government, and how, two years later, he was able to settle down to work in his new Institute for Physical Problems of the Academy of Science. He had the help of two of his technicians from Cambridge, who spent several years with him in Moscow.

Kapitza's misery in his first year, which might have seriously endangered his health, was not because he was unwilling to work for his country; he always protested that he wanted to. It was because, until the Institute was ready, there was nothing he could do. To be cut off from work was dreadful for him. If he could have returned to Cambridge, supervised the transfer of equipment to Moscow, finished off certain researches and indeed been treated like a loyal Soviet citizen, he would not have complained, or at any rate not so bitterly. But - and this is what made life intolerable - he was given no trust. People were afraid to talk to him and so he had few friends. An official was detailed to look after him, and promised much, but when Kapitza's winter clothes were posted to Moscow from Cambridge he could not even get the parcel through customs without Kapitza having to pay a huge import duty. Kapitza was denied Western newspapers, but letters to and from his wife seemed to get through, as did telephone calls. I was once in R.H. Fowler's house when a call came through from Kapitza, which lasted a very long time. Apparently he could at least do that, and trunk calls must have been cheap.

A letter from Kapitza to Rutherford in the early days of the Institute records vividly the difficulties of doing research at that time under the Soviet system:

You see Soviet industry is growing at a terrific rate and all is done to make its growing organised and well planned, so that all the system of supply and production is also well planned and organised. But the supply of the factory, which is operating according to a definite plan, must be anticipated in details at the beginning of the year, and evidently represents its demands in very large figures. Such a system is indeed quite unsuitable for the supply of laboratories. I have written and spoken to the
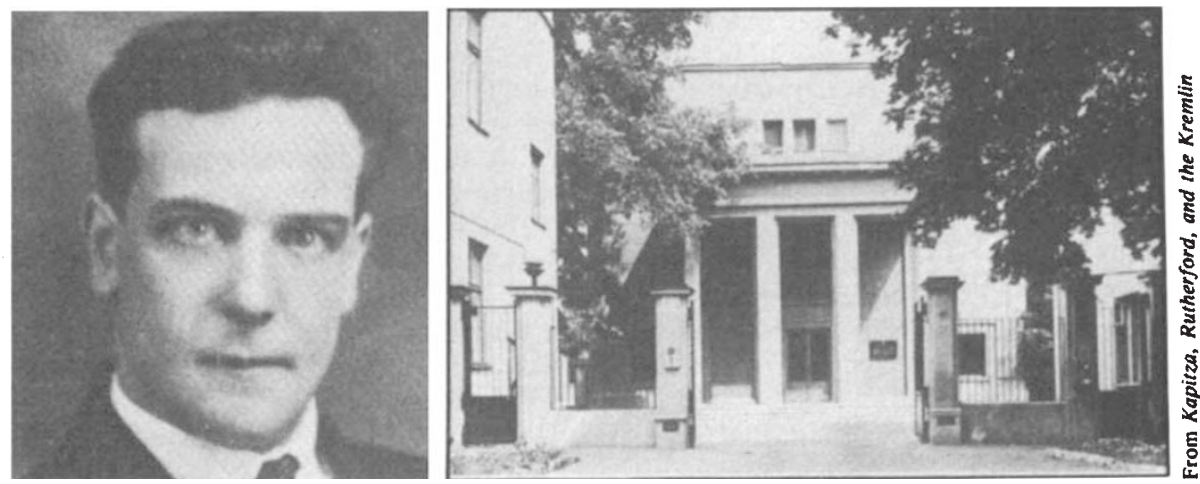

Peter Kapitza (about 1925) and the Institute for Physical Problems, Moscow. ๑)1985 Nature Publishing Group 